\title{
Observing the Influence of X-Rays on Aqueous Copper Solutions by In Situ Combined Video/XAFS/UV-Vis Spectroscopy
}

\author{
J. Gerbrand Mesu, Andrew M. Beale, Frank M. F. de Groot, Bert M. Weckhuysen \\ Inorganic Chemistry and Catalysis, Department of Chemistry, Utrecht University, Sorbonnelaan 16, 3584 CA \\ Utrecht, The Netherlands
}

\begin{abstract}
In situ video monitoring and UV-Vis spectroscopy have been used in combination with XAFS spectroscopy to study the effect of synchrotron radiation on a series of copper solutions in a micro-reactor. The samples that were investigated contained initially a mixture of $\mathrm{Cu}^{2+}$ ions and both biologically and non-biologically relevant amine ligands. It was observed that when water was used as the solvent, gas bubbles are formed under the influence of the X-ray beam. At the resultant liquid-gas interface and under certain conditions, colloidal copper nanoparticles were observed to form. This reduction process was influenced primarily by the type of the copper precursor salt $\left(\mathrm{SO}_{4}{ }^{2-}, \mathrm{NO}_{3}{ }^{-}\right.$and $\left.\mathrm{Cl}^{-}\right)$, although the ligands surrounding the copper cation and the redox potential of the copper complexes (ranging between +594 and $360 \mathrm{mV}$ ) were also observed to have some effect. Critically we show how these results illustrate the benefits of combining methods (and in particular the use of video imaging) to monitor chemical processes and for observing the influence of one technique on the measurement process. Furthermore the results give some insight into the parameters that are important in the redox-processes that occur in biological systems.
\end{abstract}

Keywords: in situ, video imaging, beam influence

PACS: $82.30 \mathrm{Vy}$

\section{INTRODUCTION}

The application of X-ray absorption spectroscopy (XAFS) to yield information on element oxidation and/or coordination state is already well established in many areas of scientific research. However the influence of X-ray photons on sample stability is not always appreciated or well understood. XAFS is, in a way, a destructive characterization technique since a photoelectron is ejected from one of the core levels of the absorber atom, leaving behind a core hole. Via different kinds of radiative decay processes the core hole in the absorber atom is filled by electrons from higher shells. This ultimately leaves an absorber atom with one electron missing in the shell with the highest energy, the valence shell. Most of the time, the electron hole is filled up by an electron from the direct environment of the absorber atom and no appreciable damage to the absorbing species occurs. If this is not the case, charging, a common problem in electron microscopy, of the specimen may occur. In general a sample can be damaged by an incident X-ray beam either directly or indirectly. The direct influence is commonly called Primary beam damage and is caused by the direct interaction of the ionizing radiation with the electrons in the object via photoelectric, Auger or Compton effects that can cause direct damage to the chemical bonds within the specimen. The formation of electrons by this primary process leads to species (such as 'energetic' electrons or free radicals) which can also react with and alter the chemical state of the specimen and hence are often considered to be part of an indirect process or secondary beam damage. The primary damage only depends on the energy of the incident radiation and the photons absorbed, whilst secondary damage depends on the properties of the specimen.[1]

With the advent of high brilliance, highly focused $\mathrm{X}$-ray beams from third generation synchrotron sources, the observation of beam damage or beam influence to various types of sample is becoming more common and poses a number of problems. Especially in circumstances where the experimental time resolution relies on increasing photon statistics rather than improvements in detector performance. Whilst a number of novel approaches have been developed in order to counter this problem it is clear that in order to fully utilize the X-ray beams now available a more fundamental understanding of the chemical processes involved in radiation damage is of paramount

CP882, X-ray Absorption Fine Structure-XAFS13 edited by B. Hedman and P. Pianetta 
importance, so that, where possible, appropriate actions can be taken.[2]

In this paper, we present insight into the influence of the effect of the X-ray beam on aqueous solutions containing copper complexes. Some of the ligands investigated included both biologically and nonbiologically relevant basic nitrogen containing ligands.[3] In addition to using in situ UV-Vis and XAFS spectroscopy to study the X-ray influence, video spectroscopy was applied in order to obtain a more detailed image into the micro-reactor as soon as the aqueous copper solutions are exposed to the X-ray beam. An additional advantage to the video imaging is that it allowed us to probe the contents of the entire cuvette rather than simply the area at focal point of the incoming light, which is typically at a maximum for the UV-Vis light in the order of a few millimeters and is therefore comparatively smaller. We believe that this study is the first of its kind where the use of a combination of real time resolved in situ video monitoring and UV-Vis spectroscopy has been used to give a detailed insight in the potential negative effects that X-ray beams may have on aqueous transition metal ion solutions. The findings may have broader implications for the field of transition metal ion catalysis as well as bio-inorganic chemistry.

\section{EXPERIMENTAL}

In addition to the various ligands used, the effect of the copper salt, the copper concentration $(20-100$ $\mathrm{mM}$ ), copper:ligand ratio (up to 1:10) and solvent were also investigated. The video/UV-Vis/ED-XAFS data were collected at the undulator beam line ID24, at the ESRF (Grenoble, France). A special designed set-up was used which was based on a setup previously used to obtain combined UV-Vis/ED-XAFS data using a quartz cuvette $(80 \mu \mathrm{l})$ mounted on a Bio-logic stopped flow module (SFM-400).[4] In this set-up either video movies or UV-Vis spectra can be collected perpendicular to the EDXAFS data. The windows in the X-ray direction are made out of vitreous carbon so as to minimize $\mathrm{X}$-ray absorption, whereas the windows perpendicular to the X-rays through which the UV-Vis and video data was collected, are made of clear quartz. An approximate X-ray photon flux of $10^{12}$ photons $/ \mathrm{s}^{-1}$ over an energy range of 8910 to $9250 \mathrm{eV}$ was focused using a curved $\mathrm{Si}(111)$ Bragg polychromator crystal to a spot size of $0.03 \times 0.2 \mathrm{~mm}^{2}$ ( $\mathrm{H} \mathrm{x} \mathrm{V}$, FWHM). The XAFS data were collected using a Peltier cooled Princeton CCD camera. The UV-Vis spectra (in the range from 200-735 $\mathrm{nm}$ ) were collected in transmission mode, perpendicular to the incident X-ray beam, using a MMS-UV1/500-1 high speed diode spectrometer equipped with optical fibers. The video movies were recorded at a speed of 25 frames/ second using a Sony Digital Hyper HAD color video camera, equipped with a high magnification focal lens.

\section{RADIOLYSIS EFFECTS ON AQUEOUS SOLUTIONS}

First, as a blank experiment, demineralized water was exposed to the X-ray beam. As soon as the sample was exposed to the X-ray beam, the formation of gas bubbles in the cuvette was observed to occur and the gas bubbles grew in size with increased exposure time. This observation indicated that the presence and growth of gas bubbles occurred as a direct result of the interaction of X-rays with the water. This process, which is commonly known as radiolysis, is known to cause fragmentation of water into a number of radical and ionic species and free electrons as seen in the equation below:

$$
\mathrm{H}_{2} \mathrm{O}+h v_{(\text {X-ray })} \rightarrow \mathrm{H}_{2} \mathrm{O}^{+}+\mathrm{e}_{(\text {dry })}^{-} \stackrel{\mathrm{H}_{2} \mathrm{O}}{\rightarrow} \mathrm{H}_{3} \mathrm{O}^{+}+\mathrm{HO}^{\bullet}+\mathrm{e}_{(\text {(aq })}^{-}
$$

The majority of these species recombine to reform water, but the radiolysis products individually also may recombine, forming new species, like $\mathrm{H}_{2}$ and $\mathrm{H}_{2} \mathrm{O}_{2}$. [5] Impurities in the water (such as the copper/ligand solutions) cause $\mathrm{H}_{2} \mathrm{O}_{2}$ to break down, releasing oxygen and hence the bubbles formed are likely to contain a mixture of $\mathrm{H}_{2}$ and $\mathrm{O}_{2}$ gases. Indeed in figure 1 we observe this effect for an aqueous solution containing $100 \mathrm{mM}$ of a $\mathrm{Cu}^{2+}$-histidine $(1: 2)$ complex at $\mathrm{pH} 6.0$ exposed to the X-ray beam. In figure 2 we show the in situ UV-Vis and XAFS spectra collected on the same sample. However, as can be seen from both figures, additional phenomena could be observed. In addition to bubble formation, colloidal copper particles formed, initially at the position where the X-ray beam is entering the cuvette, before accumulating at the bottom. It is difficult at this stage to be sure what is responsible for the reduction of the copper complex to form the colloidal metallic particles; the $\mathrm{H}_{2}$ present in the solution, or the 'hydrated electron', one of the products of the radiolysis of water. However, colloidal copper did not form every time copper solutions were exposed to an $\mathrm{X}$-ray beam. We rationalized, that this was due to the tendency of either the counter anion or the ligand to suppress the effect of the hydrated electron. In particular we observed significantly less or no colloidal copper formation when counter anions such as sulfate or nitrate were used or with bulky ligands such as terpyridine which might provide steric protection of the copper cation from the hydrated electron. 


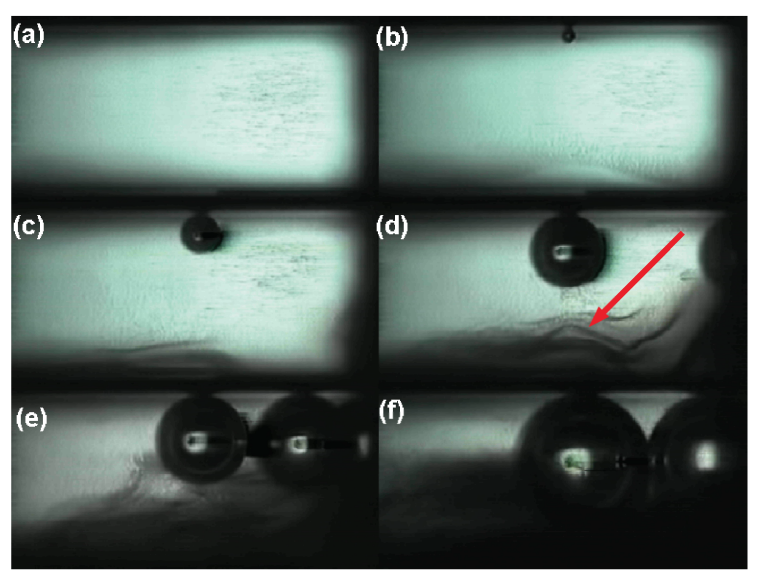

FIGURE 1. Video images illustrating the effect of the X-ray beam (enters from RHS) on aqueous $100 \mathrm{mM}$ copperhistidine (1:2) solution at $\mathrm{pH} 6.0$ as a function of time after (a): $0 \mathrm{~s},(\mathrm{~b}): 45 \mathrm{~s},(\mathrm{c}): 120 \mathrm{~s},(\mathrm{~d}): 240 \mathrm{~s}$, (e): $360 \mathrm{~s}$ and (f): 600 s. Note the formation of gas bubbles and colloidal copper (marked with an arrow) at the bottom of the microreactor.

\section{CONCLUSIONS}

In this paper we demonstrate the benefits of using a multiple spectroscopic approach to observe the effects that $\mathrm{X}$-rays have on aqueous solutions. Under the influence of X-rays, radiolysis of water occurs which results in the production of reactive species, such as the hydrated electron, which cause reduction of the copper species although it was not clear whether this was via a direct interaction with $\mathrm{Cu}^{2+}$ or through an initial interaction with the coordinating ligand. However the extent of reduction did show a dependency on the ligand type, the nature of the counter anion and possibly on the reduction potential. Based on these observations we conclude that it is difficult to study catalytic mechanisms in watercontaining systems by using X-rays, as the technique introduces new, reactive species within the system. However in this study the phenomena are exaggerated since a highly focused, high flux X-ray beam is used. But the same radiolysis products are generated at lower flux, although the effects might be less obvious. The results also show the benefits of using combined in situ spectroscopic techniques to observe and understand the processes that occur during in situ reaction and, in particular, to probe the influence that one technique (particularly those that employ high energy light beams) has on the sample stability. In addition, we have demonstrated the benefits of using real time 'video-spectroscopy' for a better understanding of the phenomena occurring in a microreactor used for studying catalytic phenomena.
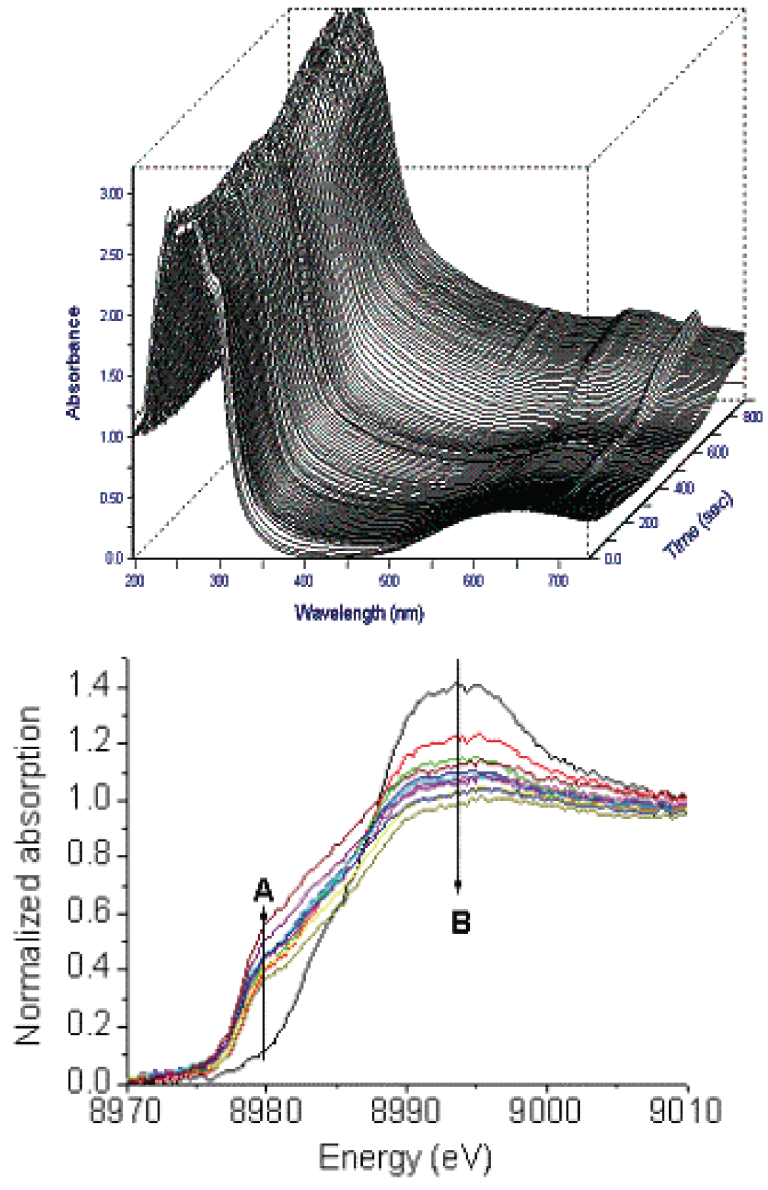

FIGURE 2. UV-Vis spectra (upper) illustrating the disappearance of the $\mathrm{d}-\mathrm{d}$ band at $650 \mathrm{~nm}$ for the initial copper histidine $(1: 2)$ aqueous solution at $\mathrm{pH}=6.0$ and XANES spectra (lower) in which arrow A indicates the evolution of a feature at $8980 \mathrm{eV}$ due to $\mathrm{Cu}(0)$ formation.

\section{REFERENCES}

1. T. Teng, K. Moffat, J. Sync. Rad., 7, 313 (2000); V. Cherezov, A. Cheng, J. M. Petit, O. Diat, M. Caffrey, Cell. Mol. Biol., 46, 1133 (2000).

2. L. M. Murphy, B. R. Dobson, M. Neu, C. R. Ramsdale, R. W. Strange, S. S. Hasnain, J. Synch. Rad., 2, 64 (1995) G. Meitzner, J. Gardea-Toffesdey, J. Parsons, S. L. Scott, E. W. Deguns, Microchem. J., 81, 61, (2005) J. Murray, E. F. Garman J. Synch. Rad.,9, 347 (2002).

3. J. G. Mesu, A. M. Beale, F. M. F. de Groot, Weckhuysen, B. M. Accepted J. Phys Chem. B.

4. M. Tromp, Developments of Time-Resolved XAFS Spectroscopy Techniques; Ph.D. Thesis, Utrecht University (2004)

5. G. Stein, (Ed.), Radiation Chemistry of Aqueous Systems, The Weizmann Science press of Israel, Jerusalem, (1968) B. Pastina, J. A. LaVerne, S. M. Pimblott, J. Phys. Chem. A, 103, 5841, (1999).

6. J. G. Mesu, A. M. J. van der Eerden, F. M. F. de Groot, B. M. Weckhuysen J. Phys. Chem. B 109, 4042 (2005) 